\title{
Detección de la intoxicación por cannabinoides sintéticos en Urgencias: cuando las pruebas toxicológicas rutinarias no bastan
}

\section{Detection of synthetic cannabinoid intoxication in the Emergency Department: when routine toxicological tests are not enough}

\author{
Francesco Dal Santo*, Ángela Velasco**,***,****, Lorena de la Fuente-Tomás**,***,****, \\ Leticia GonzÁlez-Blanco*******,****, Julia Rodríguez-Revuelta*,*****. \\ * Servicio de Salud del Principado de Asturias (SESPA), Oviedo, España. ** Área de Psiquiatría, Universidad de Oviedo, \\ Oviedo, España. *** Instituto de Investigación Sanitaria del Principado de Asturias (ISPA), Oviedo, España. **** Centro de \\ Investigación Biomédica en Red de Salud Mental (CIBERSAM), Oviedo, España.
}

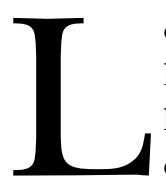
os cannabinoides sintéticos (CS) son un grupo heterogéneo de sustancias con alta afinidad para los receptores cannabinoides y representan una clase emergente de nuevas drogas, cuyo consumo ha ido expandiéndose rápidamente en los últimos años (Ford, Tai, Fantegrossi y Prather, 2017). Vendidos inicialmente a través de páginas web especializadas como alternativas legales a la marihuana en forma de mezcla de hierbas, bajo los nombres de "Spice" (Europa) o "K2" (EEUU), son objeto de vigilancia por parte del Observatorio Europeo de las Drogas y las Toxicomanías (EMCDDA) desde 2008, cuando se demostró la presencia en estos productos del CS JWH-018 (Observatorio Europeo de las Drogas y las Toxicomanías, 2017). La continua modificación y manipulación de estos compuestos por parte de los laboratorios ilegales ha acelerado notablemente la introducción de nuevas moléculas en el mercado (Ford et al., 2017): en diciembre de 2016 se habían notificado 169 CS al EMCDDA (Observatorio Europeo de las Drogas y las Toxicomanías, 2017), detectando sustancias progresivamente más potentes (Adams et al., 2017).

El aumento del consumo de sustancias psicotrópicas ilegales, especialmente el cannabis, entre los más jóvenes (Blasco-Fontecilla, 2018), y el frecuente uso desadaptativo de internet en esta población (Golpe, Gómez, Braña, Varela y Rial, 2017), podrían facilitar la adquisición y consumo de CS ya desde la adolescencia: en la encuesta ESTUDES 2016 el $0.9 \%$ de los estudiantes de 14-18 años declaraba haber probado el "Spice" al menos una vez en la vida (Plan Nacional sobre Drogas, 2016).
Los productos cannabinoides sintéticos poseen elevada afinidad para el receptor CB1 en comparación con el delta-9-THC: a diferencia de los derivados cannábicos herbales, que contienen diferentes moléculas con potencia psicoactiva variable (Casajuana Köguel, López-Pelayo, Balcells-Olivero, Colom y Gual, 2018), los CS actúan como agonistas puros del receptor (Ford et al., 2017). Existen evidencias experimentales que indican que los CS también actúan sobre receptores no-cannabinoides, como el receptor 5-HT2B o receptores dopaminérgicos (Adams et al., 2017). Además, los CS carecen en su composición de cannabidiol, presente en el cannabis herbal y capaz de ejercer efectos antipsicóticos, moderando la acción del delta-9THC (Rowley et al., 2017). Estas características se traducen en un mayor efecto psicoactivo, además de incrementar la frecuencia y gravedad de los efectos secundarios.

Entre los síntomas psiquiátricos por intoxicación pueden observarse ansiedad, agitación, alucinaciones, confusión, amnesia, delirios paranoides, conductas extrañas, heteroagresividad e ideación autolítica. A nivel somático pueden provocar taquicardia, hipertensión, somnolencia, deterioro del nivel de consciencia, vértigo, parestesias, crisis epilépticas, infarto agudo de miocardio y accidentes cerebrovasculares, llegando incluso hasta la muerte en algunos casos (Tournebize, Gibaja y Kahn, 2017). El consumo concomitante de otras sustancias suele asociarse a una mayor gravedad de la clínica (Rowley et al., 2017).

Dada la variedad y la naturaleza de las sustancias disponibles, se requieren procedimientos como la cromatografía de gases o el espectrómetro de masas para su deter-

Recibido: Diciembre 2018; Aceptado: Enero 2019.

Enviar correspondencia a: Francesco Dal Santo. Departamento de Psiquiatría, Facultad de Medicina.

Av. De Julián Clavería nº, $3^{a}$ planta. CP: 33006. Oviedo, Asturias, España.

E-mail: frdalsanto@gmail.com. 
minación analítica, con importantes limitaciones para la detección de los CS en la práctica clínica habitual. Algunos usuarios de cannabis podrían optar por los CS para eludir la positividad de las pruebas (Rowley et al., 2017).

Por este motivo y por las manifestaciones clínicas inespecíficas, ante la normalidad en las pruebas toxicológicas de rutina, los cuadros de intoxicación por CS pueden llegar a confundirse con otras patologías psiquiátricas o somáticas.

En la actualidad, se asiste a un aumento de consultas a los Servicios de Urgencias por intoxicación por CS (Tournebize et al., 2017). Se han descrito casos de intoxicación masiva: en New York, en Julio de 2016, se atendieron 33 personas en el contexto de la notoria "epidemia zombie” provocada por la exposición al CS AMB-FUBINACA (Adams et al., 2017), despertando las alarmas entre la población, los medios de comunicación y los profesionales sanitarios.

Estudios previos destacan que los consumidores de CS tienen un riesgo hasta 30 veces mayor de acudir a Urgencias por los efectos de la intoxicación aguda en comparación con los consumidores de cannabis natural (Winstock, Lynskey, Borschmann y Waldron, 2015). La mayoría de los pacientes suele recibir tratamiento con fluidoterapia intravenosa, fármacos sedantes y antieméticos, aunque pueden hacerse necesarias medidas como sedación o intubación $\mathrm{y}$, hasta en un cuarto de los casos, el ingreso hospitalario (Rowley et al., 2017).

En relación con lo anteriormente expuesto, nos gustaría destacar la especial relevancia que adquiere en la actualidad la formación de los profesionales implicados en la atención en los Servicios de Urgencias sobre las nuevas sustancias psicoactivas. La creciente difusión de estas drogas, especialmente entre la población joven, implica la necesidad de conocimientos actualizados para poder averiguar un posible cuadro de intoxicación por CS, establecer un correcto diagnóstico diferencial y permitir la pronta instauración del tratamiento más adecuado. Sin olvidarnos que, ante la negatividad de las pruebas toxicológicas habituales, la atenta observación de las manifestaciones clínica sigue constituyendo la herramienta más eficaz a nuestra disposición.

\section{Conflictos de intereses}

Los autores de este artículo declaran que no existe conflicto de interés.

\section{Bibliografía}

Adams, A. J., Banister, S. D., Irizarry, L., Trecki, J., Schwartz, M. y Gerona, R. (2017). «Zombie» Outbreak Caused by the Synthetic Cannabinoid AMB-FUBINACA in New York. The New England Journal of Medicine, 376, 235242. doi:10.1056/NEJMoa1610300.

Blasco-Fontecilla, H. (2018). Postmodernity, addictive societies, cannabis and suicidal behaviour: Towards a brave new world? Adicciones, 30, 3-8. doi:10.20882/adicciones. 1104.

Casajuana Köguel, C., López-Pelayo, H., Balcells-Olivero, M. M., Colom, J. y Gual, A. (2018). Psychoactive constituents of cannabis and their clinical implications: a systematic review. Adicciones, 30, 140-151. doi:10.20882/ adicciones.858.

Ford, B. M., Tai, S., Fantegrossi, W. E. y Prather, P. L. (2017). Synthetic Pot: Not Your Grandfather's Marijuana. Trends in Pharmacological Sciences, 38, 257-276. doi:10.1016/j.tips.2016.12.003.

Golpe, S., Gómez, P., Braña, T., Varela, J. y Rial, A. (2017). The relationship between consumption of alcohol and other drugs and problematic Internet use among adolescents. Adicciones, 29, 268-277. doi:10.20882/adicciones.959.

Observatorio Europeo de las Drogas y las Toxicomanías. (2017). Cannabinoides sintéticos en Europa. Recuperado de http://www.emcdda.europa.eu/topics/pods/ synthetic-cannabinoids_es.

Plan Nacional sobre Drogas. (2016). Encuesta sobre uso de drogas en Enseñanzas Secundarias en España (ESTUDES) 2016. Recuperado de http://www.pnsd.mscbs. gob.es/profesionales/sistemasInformacion/sistemaInformacion/encuestas_ESTUDES.htm.

Rowley, E., Benson, D., Tiffee, A., Hockensmith, A., Zeng, H., Jones, G. N. y Musso, M. W. (2017). Clinical and financial implications of emergency department visits for synthetic marijuana. The American Journal of Emergency Medicine, 35, 1506-1509. doi:10.1016/j. ajem.2017.04.044.

Tournebize, J., Gibaja, V. y Kahn, J. P. (2017). Acute effects of synthetic cannabinoids: Update 2015. Substance Abuse, 38, 344-366. doi:10.1080/08897077.2016.1219438.

Winstock, A., Lynskey, M., Borschmann, R. y Waldron, J. (2015). Risk of emergency medical treatment following consumption of cannabis or synthetic cannabinoids in a large global sample. Journal of Psychopharmacology, 29, 698-703. doi:10.1177/0269881115574493. 